\title{
¿Sin libertad hay ciudadanía? El ejercicio de la participación formal de la ciudadanía de las personas privadas de libertad en Costa Rica
} is There Citizenship Without Freedom? The Exercise of Formal Citizenship Participation of Persons Deprived of Liberty in Costa Rica

\author{
Jesús Sáenz Solís ${ }^{1}$ \\ Dioni Céspedes Bolaños ${ }^{2}$ \\ Sebastián Barquero Zúñiga ${ }^{3}$
}

Universidad de Costa Rica

Costa Rica

\section{Resumen}

El presente artículo tiene como objetivo entender cómo se practica la participación ciudadana desde la privación de libertad y cuáles son los mecanismos formales identificados por el ordenamiento jurídico, así como sus limitaciones y alcances. Se propone reconstruir los principales elementos que muestran la evolución del acceso a la participación ciudadana formal de las personas privadas de libertad en Costa Rica. La metodología utilizada fue el análisis de datos de las elecciones presidenciales desde que se permitió el voto de dicha población (1998) y hasta el año 2018. Además, se muestran datos de otro tipo de actividades, como el referendum del año 2007 y el uso de herramientas jurídicas como los amparos electorales presentados en función de garantizar los derechos

1 Politólogo de la Universidad de Costa Rica, especialista en Políticas Públicas para la Igualdad en América Latina de CLASCO y de FLACSO sede Brasil, Investigador asociado del Informe Estado de la Justica del Programa Estado de la Nación y consultor.

Correo electrónico: jsaenz@estadonacion.or.cr

2 Politóloga. Correo electrónico: dionicespedes@gmail. com

3 Politólogo. Correo electrónico: sebasbz21@ gmail.com 
políticos en las cárceles. Por otro lado, la discusión gira en torno al ejercicio de la participación ciudadana como uno de los temas que cuenta con un mayor acervo investigativo en las Ciencias Sociales, el cual, sin embargo, ha sido poco explorado en el contexto de poblaciones en altas condiciones de vulnerabilidad como lo son las personas privadas de libertad. Por lo tanto, entre los hallazgos se muestran bajos niveles de participación y se presupone la existencia de importantes limitaciones no en el acceso como tal, sino en los elementos que permiten hacer efectivos los derechos. Se concluye que las garantías que poseen la población penal en esta materia constituyen un desafío para las autoridades, en términos del ejercicio de la ciudadanía y el respaldo normativo nacional e internacional.

Palabras clave: participación ciudadana; prisión; voto; democracia; derechos políticos; privados de libertad.

\begin{abstract}
This article aims to understand how citizen participation is practiced from the point of view of deprivation of liberty and what are the formal mechanisms identified by the legal system, as well as their limitations and scope. The proposal is to reconstruct the main elements that show the evolution of access to formal citizen participation of persons deprived of liberty in Costa Rica. The methodology implemented was the analysis of data from the presidential elections. These considered data were from 1998 (when the population was allowed to vote) until 2018. Besides, data from other types of activities, such as the 2007 referendum and the use of legal tools, such as the electoral appeals filed to guarantee political rights in prisons, were examined. On the other hand, the discussion revolves around the exercise of citizen participation as one of the most studied subjects in the Social Sciences, which, however, has been little explored in the context of populations in high conditions of vulnerability, such as persons deprived of liberty. Therefore, the findings show low levels of participation and presuppose the existence of important limitations, not in access as such, but in the elements that allow the exercise of rights. It is concluded that the guarantees available to the prison population in this area constitute a challenge for the authorities in terms of the exercise of citizenship and national and international normative support.
\end{abstract}

Keywords: citizen participation; prison; vote; democracy; political rights; deprived of liberty.

\title{
1. Introducción
}

La participación ciudadana, como el estudio del tema de investigación politológica, ha aumentado en las academias costarricenses e internacionales (Font, 2013; 
Pignataro y Cascante, 2018). Sin embargo, los análisis generalmente se restringen a la participación político electoral, lo cual ha implicado una menor atención a otras formas de participación, como la organización comunitaria o las acciones de visibilización de necesidades de poblaciones en condición de vulnerabilidad. A esto se debe agregar que el estudio de la participación ciudadana enfocado en poblaciones vulnerabilizadas, como la población privada de libertad en Costa Rica, es prácticamente inexistente. Por lo tanto, es importante estudiar, desde las Ciencias Sociales, las diferentes ramas de participación ciudadana para las poblaciones vulnerabilizadas y excluidas socialmente con el fin de fortalecer del sistema democrático, que parte del principio de representación de todas las personas sin exclusión.

A partir de la visibilización de la diversidad de factores que inciden en las diferentes formas en que se construye la participación ciudadana en el sistema democrático, se propone investigar el ejercicio de la participación ciudadana de personas privadas de libertad en Costa Rica en los más recientes procesos electorales. Esto se da en el contexto de un alarmante incremento de esta población en el país: entre el 2005 y el 2015 la cantidad de personas en privación de libertad aumentó en un $60 \%$ (Estado de la Justicia, 2017).

A la fecha, carecemos de información relevante y sistematizada del ejercicio de derechos políticos bajo condiciones de privación de libertad, por lo que este trabajo resulta de vital importancia para brindar un panorama general, en términos del acceso de la población penal al voto. Además, los insumos de esta investigación pueden generar conocimiento valioso para la toma de decisiones y generación de políticas públicas concernientes a la población privada de libertad, con el fin de garantizar más y mejores mecanismos de participación ciudadana desde la institucionalidad de un Estado de Derecho.

En esta investigación se estudia cómo se practica la participación ciudadana desde la privación de libertad y cuáles son los mecanismos formales identificados por el ordenamiento jurídico, así como sus limitaciones y alcances. Para ello se realiza una descripción de los mecanismos formales de participación ciudadana de las personas privadas de libertad en Costa Rica, a partir de una revisión de algunos elementos del marco jurídico costarricense en materia de derechos políticos, reseña de los recursos de amparo electoral presentados por privados de libertad, estadísticas de los principales procesos electorales. Se hace referencia a dos centros penitenciarios de interés como lo son la UAI Reynaldo Villalobos y el CAI Jorge Arturo Mora. También se incluye información sobre el voto en el referéndum del año 2007. 


\section{La participación ciudadana en privación de libertad}

Se entiende por privación de libertad "cualquier forma de detención, encarcelamiento, institucionalización o custodia de una persona, por razones de asistencia humanitaria, tratamiento, tutela, protección, o por delitos e infracciones a la ley" (Corte Interamericana de Derechos Humanos, 2008).

Por su parte, se considera que el ejercicio de la participación ciudadana es el resultado de acciones colectivas e individuales que tienden a la construcción de espacios sociales, económicos, políticos, jurídicos y culturales que permiten presentarse a la sociedad como individuos o colectivos con derechos y obligaciones.

Una definición más operacional sería:

(...) cualquier acción de los ciudadanos dirigida a influir en el proceso político y en sus resultados. Estas acciones pueden orientarse a la elección de los cargos públicos (más allá de los elegidos a través de la consulta popular durante el procedimiento electoral); a la formulación, elaboración y aplicación de políticas públicas que éstos (sic) llevan a cabo; o a la acción de otros actores políticos relevantes. (Instituto Interamericano de Derechos Humanos, 2011, p. 27)

Asimismo, es fundamental comprender que los derechos humanos asociados a la participación ciudadana deben observarse de forma crítica como una "apertura a procesos de lucha por la dignidad humana" (Herrera, 2008, p. 15), lo cual se relaciona con el ejercicio de la participación ciudadana como mecanismo para el reconocimiento de condiciones dignas y necesarias de la población privada de libertad.

\subsection{La participación ciudadana en centros penitenciarios costarricenses}

En términos demográficos y según las estadísticas del Ministerio de Justicia y Paz (2017), nos encontramos con que la población penal en Costa Rica para el mes de noviembre de 2017 era 36291 personas. De estas, 13983 se encontraban bajo el internamiento que establece el código penal, es decir, en condición de privación de libertad y habitaban alguno de los 19 centros penales a lo largo del país. Dentro de esta población existe una marcada diferencia de género, en tanto se encuentran privadas de libertad 550 mujeres, mientras que la cantidad de hombres es de 13 438. Esta es la razón por la que existe un único centro penitenciario (Centro de Atención Institucional Vilma Curling Rivera) exclusivo para mujeres, dos mixtos (hombres y mujeres) y 16 centros exclusivos para hombres a lo largo del país. 
Para efectos de esta investigación, es importante señalar que existe un 25,30 \% de la población privada de libertad que está en condiciones de vulnerabilidad (Ministerio de Justicia y Paz (MJP), 2017), como lo son personas afrodescendientes, población LGBTI, personas adultas mayores, extranjeros, indígenas y personas con discapacidad. Existen otras condiciones socioeconómicas que pueden incrementar la vulnerabilidad, como por ejemplo el $82,3 \%$ de la población privada de libertad posee secundaria incompleta o anteriores (MJP, 2017). Así pues, esta población presenta varias condiciones intervinientes dentro de su condición de exclusión social, económica y política, además de su condición de privación de libertad.

Por otra parte, es fundamental destacar que Costa Rica ha logrado asegurar una serie de obligaciones jurídicas que facilitan el cumplimiento de los derechos de las personas privadas de libertad, tanto desde la normativa interna como por medio de convenios internacionales. Sin embargo, a pesar de la existencia de este marco normativo, aún existe una gran carencia con respecto a la garantía del ejercicio de la participación ciudadana de esta población. Como muestra de lo anterior, fue hasta el año 1998 que las personas privadas de libertad pudieron ejercer el derecho al voto a través de la Ley 7653 del año 1996. Según Sobrado (2006), para el grueso de la población no hay condiciones óptimas y existen limitaciones en el acceso a la información para el ejercicio de este derecho, lo que se ve reflejado en los bajos niveles de participación y acceso a otros mecanismos de participación ciudadana.

Asimismo, el país ratificó en 1998 el conjunto de principios para la protección de todas las personas sometidas a cualquier forma de detención o prisión, en 1997 las "Reglas mínimas para el tratamiento de los reclusos", en el 2008 los "Principios y buenas prácticas sobre la protección de las personas privadas de libertad en las Américas" y en 1969 el "Pacto de San José". No obstante, únicamente este último considera, de forma ambigua y escueta, el ejercicio de la participación ciudadana, mientras los demás abarcan cuestiones concernientes a otras áreas, por lo que las garantías de los derechos políticos no se encuentran dentro del marco tutelado.

Sobrado (2006) plantea un análisis descriptivo sobre la experiencia de acceso a la participación político electoral de la población privada de libertad en los procesos nacionales del 2002 y el 2006. De igual manera, detalla el comportamiento del voto y la normativa en esta materia para asegurar dicha garantía desde su aprobación y regulación.

Dicho estudio es fundamental para el presente escrito, pues brinda un panorama de las asimetrías existentes en relación al ejercicio de participación ciudadana de 
la población privada de libertad. El autor analiza el comportamiento del voto de esta población y señala las grandes diferencias que existen en comparación con el resto de la ciudadanía. Asimismo, concluye con la necesidad de que futuros procesos de investigación académica detallen en las condiciones que limitan el ejercicio de este mecanismo para las personas privadas de libertad en nuestro país. Por último, plantea que deben considerarse las amplias dificultades de esta población para acceder a la información político electoral relevante, lo que el autor denomina "equiparación de las condiciones del voto" (p.17), con el fin de ejercer un voto informado.

\section{Mecanismos formales de participación ciudadana de las personas privadas de libertad}

Los mecanismos formales son aquellos que se encuentran en el orden jurídico establecido y son recursos tangibles que ofrece el Estado para hacer uso de la participación ciudadana como un derecho y como un deber. El Instituto de Formación y Estudios en Democracia (IFED) del Tribunal Supremo de Elecciones (TSE) señala que la participación ciudadana se expresa en ocho tipos de derechos. Estos son:

a) Elegir y ser electo.

b) Informarse y opinar sobre asuntos públicos.

c) Participar y pronunciarse en plebiscitos, referendos y consultas populares.

d) Reunirse y asociarse y organizarse para fines legales.

e) Pedir y obtener información.

f) Crear partidos y movimientos políticos.

g) Presentar iniciativas de ley.

h) Participar en los cabildos.

En el caso de estos derechos, al ser meramente electorales, existen mecanismos que permiten la tutela efectiva que ofrece la justicia electoral, positivizados en el ordenamiento jurídico costarricense. Estos son: el recurso de amparo, el recurso de apelación electoral, demanda de nulidad relativa a resultados electorales, cancelación de credenciales, denuncia por parcialidad o beligerancia política y los acuerdos de nulidad partidarios.

En un segundo nivel, se encuentran los mecanismos institucionales para la participación. Estos, han sido diseñados institucionalmente para reflejar los aportes de la ciudadanía costarricense y están regulados por normas y leyes. Se pueden 
nombrar: las elecciones de representantes, partidos políticos, la oficina de iniciativa popular y el referéndum. También existen otros mecanismos institucionales de carácter especializado como: las contralorías de servicios, audiencias de la Autoridad Reguladora de los Servicios Públicos (ARESEP), la Defensoría de los Habitantes y la defensoría del consumidor.

Los mecanismos a escala cantonal y distrital son los que están normados en el artículo 5 del Código Municipal de la Ley 7794 (Asamblea Legislativa de la República, 1998) que expresa la obligación que tienen las municipalidades de fomentar "la participación activa, consciente y democrática del pueblo en las decisiones del gobierno local". Los principales recursos de este tipo son: el cabildo, el referendo, el plebiscito, juntas administrativas y educativas de los centros de enseñanza pública, comités cantonales de deportes, comités cantonales de la persona joven, centros agrícolas cantonales y las asociaciones de desarrollo comunal. Los mecanismos formales de participación ciudadana acá explicitados por Castro y Sibaja (2016) cumplen con lo que el enfoque neoinstitucional llama un papel relevante dentro de la sociedad, por eso es que se regulan. El desempeño de estos mecanismos se observa a partir "de la interdependencia en el medio ambiente en que operan y de la interpretación de la situación en que se encuentran los actores" (Vargas, 2008).

Por otra parte, las perspectivas teóricas críticas sobre la democracia liberal deben permitir comprender la participación ciudadana y su ejercicio en el marco de la cuestión penal y la privación de libertad. Por lo tanto, si bien es cierto que existe una gran cantidad de normativas que les garantizan a las personas privadas de libertad sus derechos en el ámbito de la participación, existen también limitaciones para su comprensión en sentido amplio. Esto no solo se observa a través de la positivización de los derechos humanos de esta población, sino a partir de las prácticas y discursos que existen en el centro penitenciario sobre la participación ciudadana de las personas privadas de libertad y que deberán ser abordados en otras investigaciones.

\subsection{Sufragio en elecciones nacionales}

Durante los últimos 20 años, la población privada de libertad ha ejercido el sufragio como uno de los mecanismos formales de participación ciudadana. La evidencia refleja que de 1998 al 2014 el padrón electoral en cárceles ha crecido en un $400 \%$, mientras que los votos efectivos de las personas privadas de libertad han crecido en un $225 \%$. 
Para el 2014, el Tribunal Supremo de Elecciones (TSE) tenía contabilizado en el padrón electoral para las elecciones presidenciales y legislativas un total de 8713 personas privadas de libertad. Para el 2018, se dio un crecimiento del $10 \%$ (9575). Por esto, el TSE debió a abrir seis juntas receptoras de votos más que la elección anterior.

Sin embargo, este crecimiento dentro del padrón electoral debe analizarse en términos de la materialización de un aumento en el ejercicio de derechos electorales por parte de las personas privadas de libertad. En ese sentido, la figura 1 muestra cómo, si bien es cierto ha aumentado la cantidad de electores, a partir del primer proceso electoral en que se facilitó el acceso a las personas privadas de libertad, ha existido una tendencia hacia la baja en la participación.

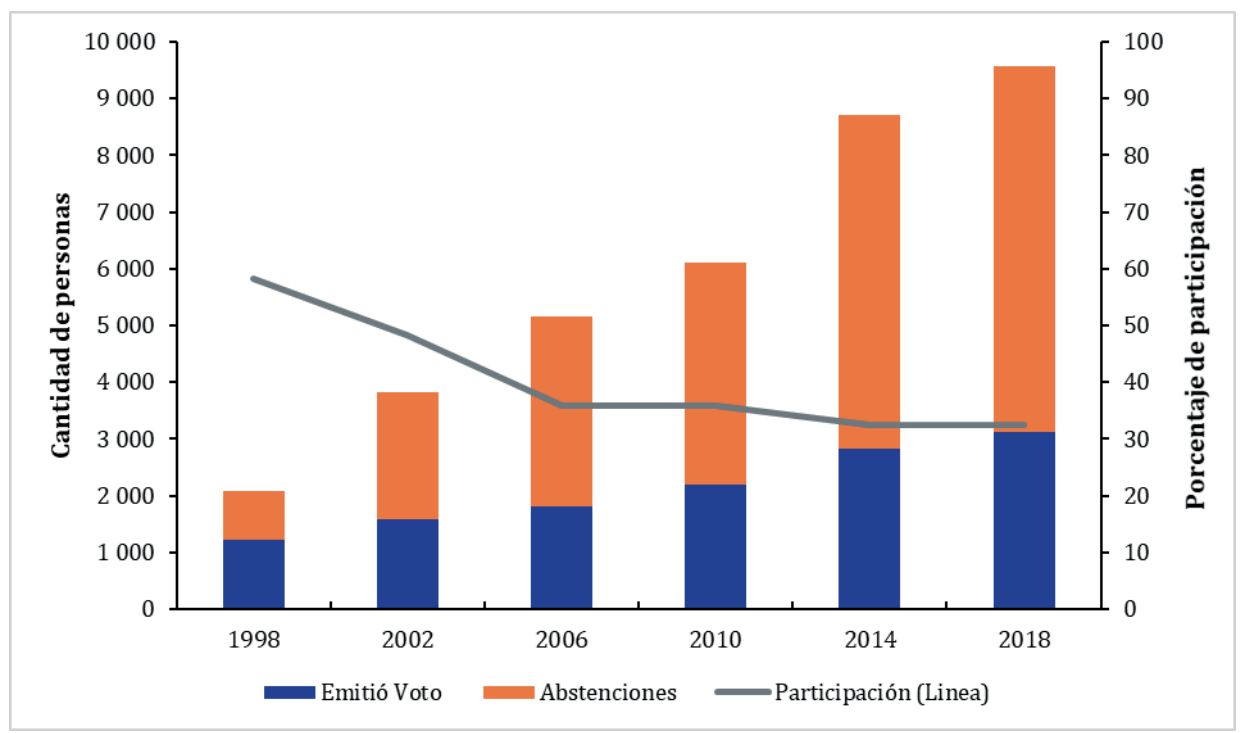

Figura 1: Participación electoral en elecciones presidenciales por personas privadas de libertad en Costa Rica.

Fuente: Elaboración propia según datos del Tribunal Supremo de Elecciones (TSE), 2018.

En ese sentido, se logra evidenciar un estancamiento en las cifras a partir del 2006, ya que desde entonces la participación de tipo electoral no ha logrado modificarse en más de 4 puntos porcentuales en los últimos 12 años. Es necesario destacar que estos porcentajes se encuentran considerablemente más bajos que los promedios nacionales, pues no alcanzan el $50 \%$ de la cifra de participación de todo el país. 
Para efectos de la presente investigación, se hace énfasis de los datos obtenidos de dos centros penitenciarios específicos: el Centro de Atención Institucional (CAI) Jorge Arturo Mora conocido como "La Reforma" y la Unidad de Atención Integral Reynaldo Villalobos. Así se logran observar posibles diferencias infraestructurales, culturales, discursivas y otras que viven las personas privadas de libertad que se encuentran en dichos centros. Estos corresponden a dos modelos de prisionalización distintos, donde en uno (CAI Jorge Arturo Mora) se experimentan condiciones de deterioro notables en infraestructura, más hacinamiento y menor atención técnica en comparación a la UIA Reynaldo Villalobos que cuenta con instalaciones más recientes y una cultura de convivencia y socialización distintas.

Para las elecciones del 2018, ambos centros se encuentran en funcionamiento, lo que permite poder obtener una perspectiva comparativa entre ellos, ya que la UIA Reynaldo Villalobos se inaugura en el 2017. La figura 2 muestra el porcentaje de participación electoral durante las elecciones presidenciales del 2018 en todos los centros penitenciarios.

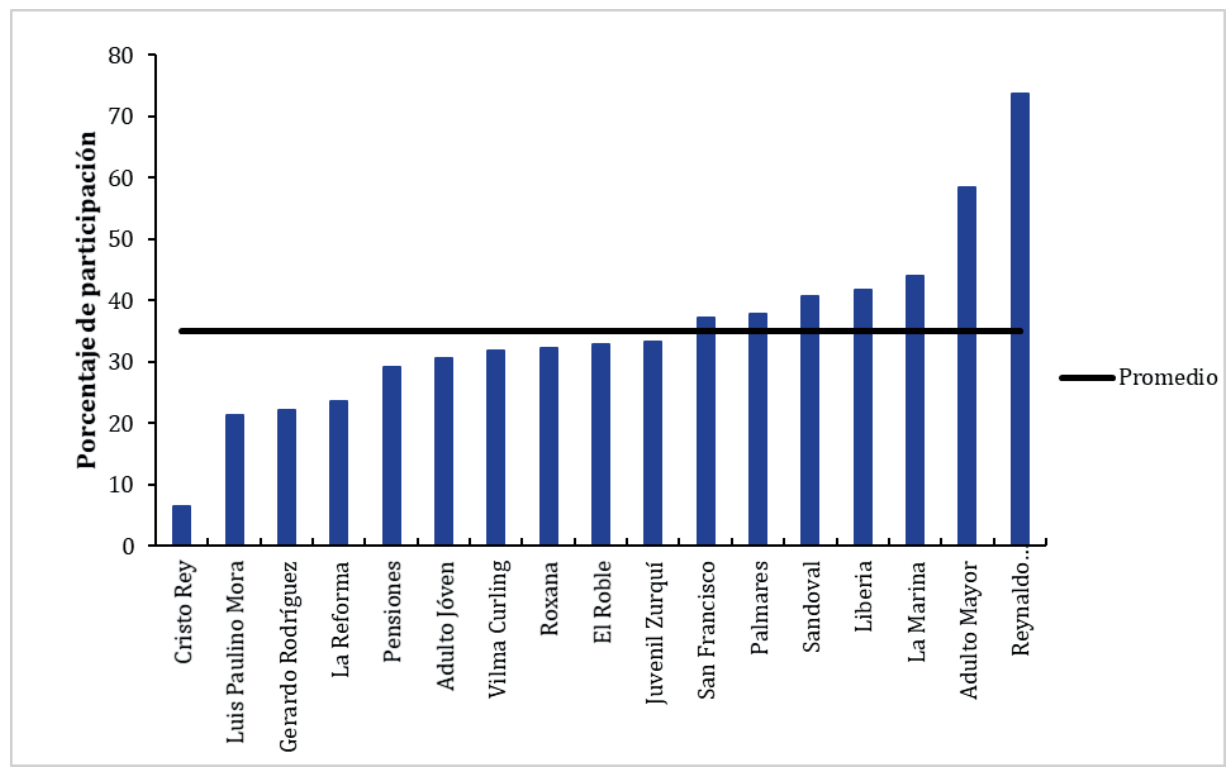

Figura 2: Participación electoral en elecciones presidenciales del 2018 por centro penitenciario.

Fuente: Elaboración propia según datos del TSE. 
Los datos seleccionados muestran como en la UAI Reynaldo Villalobos el porcentaje de participación es aproximadamente el triple del registrado en el CAI Jorge Arturo Mora. Asimismo, poseen un porcentaje que es considerablemente superior al total del porcentaje de participación en centros penitenciarios, incluso la UAI según datos del TSE fue el centro penitenciario que consignó una mayor participación electoral a nivel nacional para las elecciones presidenciales del 2018.

La información suministrada permite realizar un análisis riguroso dentro de agendas de investigación futuras sobre las causas que favorecen al surgimiento de diferencias tan relevantes. Como respuesta inicial, se plantea que las personas privadas de libertad se encuentran en un permanente proceso de interacción con el sistema político al encontrarse dentro de un centro penal. En tanto el sector estatal cumpla en condiciones más dignas el ejercicio de su poder punitivo, puede ocasionar un interés mayor de incorporarse dentro de los procesos de ejercicio de derechos políticos en términos formales.

En el caso de las personas que se encuentran en centros penitenciarios con condiciones mucho más desfavorables y en las cuales las implicaciones de violencia estructural ejercida por el poder punitivo del Estado son mayores, se pueden generar diferencias con otros centros. En ese sentido, las personas que sufren más y que reciben menor atención por parte de las instituciones no buscarán legitimar y participar de un sistema político "democrático" del cual se sienten excluidos.

Como elemento adicional es relevante analizar estas condiciones a partir de la distribución de estos votos entre todas las personas candidatas participantes dentro del proceso electoral. En primer lugar, el figura 3 muestra los datos a nivel nacional para el 2018, distribuidos por partido político en centros penitenciarios. 


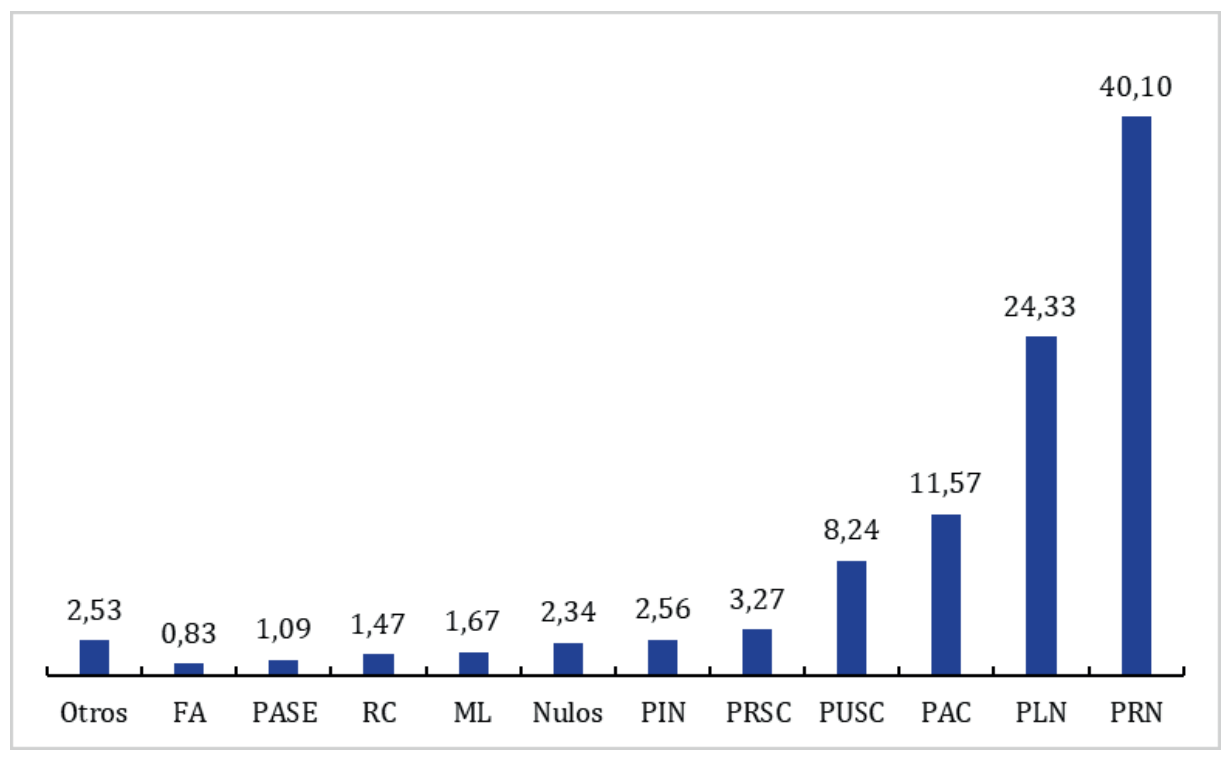

Figura 3: Porcentaje de votos obtenidos por partido político en las elecciones presidenciales 2018 en centros penitenciarios en Costa Rica.

Fuente: Elaboración propia según datos del TSE (2018).

El partido que logra una mayor cantidad de votos por parte de la población penal es el Partido Restauración Nacional con un 40,7 \% de los votos totales. Interesa indicar que esta misma agrupación política durante el proceso electoral afirmaba: "como consecuencias de un sistema de justicia alcahuete e hiperflexible, que hace que muchas lacras de la sociedad cometan los delitos y se pavoneen por nuestras calles como una afrenta del sistema para con las víctimas" (Pignatario y Cascante, 2018). Es decir, se parte de una perspectiva punitivista que permite y persigue la profundización la violencia estructural, ejercida por las instituciones penitenciarias.

Las visiones expuestas por agrupaciones más conservadoras se anteponen por ejemplo, al desarrollo de un modelo garantista para la tutela de los derechos de las personas privadas de libertad, que tuvo avances importantes durante el gobierno del Partido Acción Ciudadana (PAC) en el período 2014-2018. A pesar de esto, el PAC logra únicamente un $11,7 \%$ de los votos totales obtenidos.

Estas dos consideraciones llevan a una gran interrogante: ¿qué motivaciones conducen a las personas privadas de libertad a favorecer candidatos con una orientación punitivista y que podría afectar sus condiciones de vida? A pesar de ser 
una pregunta pendiente de respuesta certera, un elemento por considerar es la influencia de los discursos de tipo religioso en el contexto de la discusión de los asuntos públicos y definición de las motivaciones para el ejercicio del sufragio. Lo anterior se antepone a las visiones más conservadoras del análisis político como las teorías de la elección racional.

Es fundamental para efectos del análisis que se logre verificar si las diferencias ubicadas dentro de los porcentajes de participación se logran ubicar también dentro de cada centro. Asimismo, se toma en cuenta la distribución nacional de votos por partido y se estima conocer si sus tendencias se mantienen de forma homogénea entre centros. La figura 4 muestra los datos recopilados de la UAI Reynaldo Villalobos.

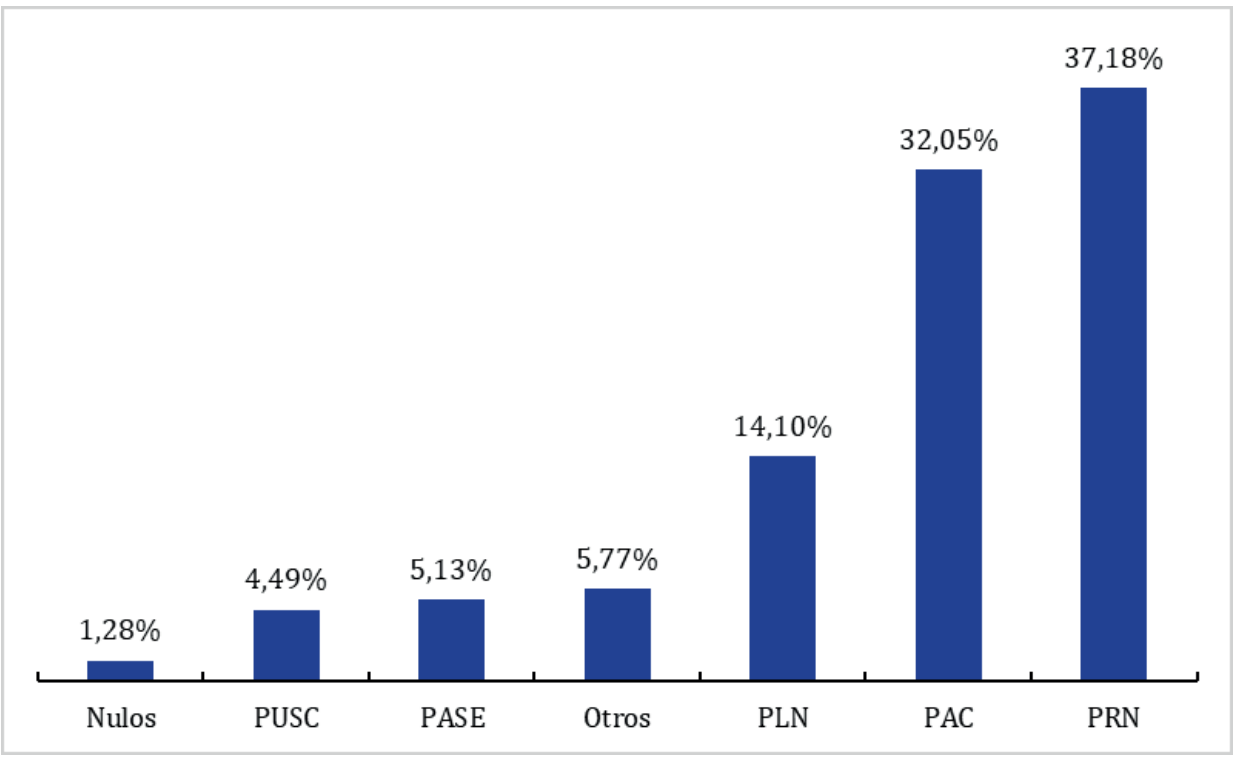

Figura 4: Porcentaje de votos obtenidos por partido político en las elecciones presidenciales 2018 en la UAI Reynaldo Villalobos.

Fuente: Elaboración propia según datos del TSE (2018).

Los datos obtenidos muestran que, a diferencia de la tendencia a nivel nacional, existen pocas diferencias en los niveles de votación obtenidos por el Partido Acción Ciudadana y el Partido Restauración Nacional. En este caso particular, se podría tomar como elemento de análisis el hecho de que este centro penitenciario fue inaugurado dentro de la administración 2014-2018. En ese sentido, la inauguración, el mantenimiento y el traslado de las personas al espacio representa 
un mejoramiento en sus condiciones materiales de vida se podría traducir en un mayor apoyo al partido de gobierno.

Sin embargo, en la figura 5 se indica que un centro penitenciario como el CAI Jorge Arturo Mora las motivaciones para el ejercicio del voto tienden a fluctuar. En ese sentido, se observa cómo se asemeja de forma considerable a los porcentajes totales encontrado a nivel de todo el país en centros penitenciarios.

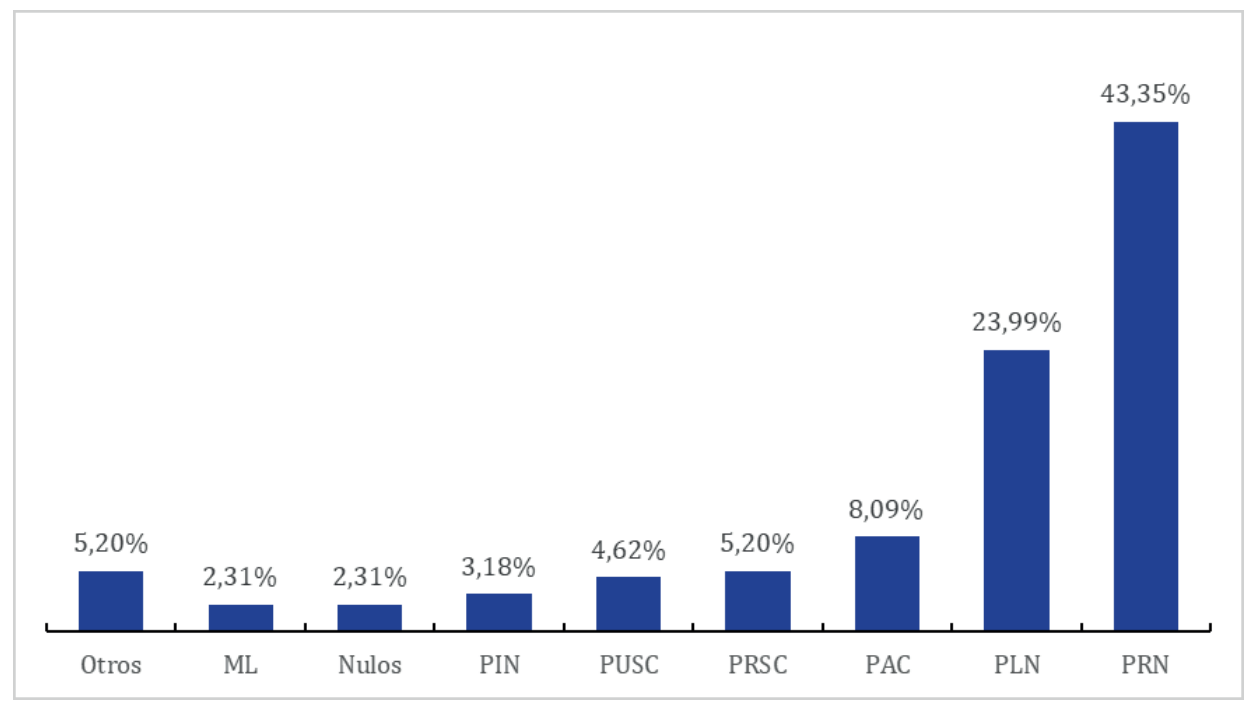

Figura 5: Porcentaje de votos obtenidos por partido político en las elecciones presidenciales 2018 en el CAI Jorge Arturo Mora.

Fuente: Elaboración propia según datos del TSE.

En el caso del CAI Jorge Arturo Mora, corresponde a una serie de ámbitos elaborados a partir de un sistema mucho más tradicional. Muchos espacios para sus condiciones de vida y convivencia no están diseñados para facilitar el cumplimiento de los derechos humanos y la generación de condiciones adecuadas. Se parte de que estas condiciones de abandono podrían generar una menor afinidad y más bien una distancia considerable con los operadores responsables de la política penitenciaria.

\subsection{Referéndum}

En lo que se refiere al referéndum del 2007 sobre el Tratado de Libre Comercio entre República Dominicana, Centroamérica y Estados Unidos, la participación 
electoral estuvo por debajo del $30 \%$. El resultado de la consulta no se alejó de las tendencias nacionales, donde el SÍ obtuvo un $52 \%$ y el NO un $48 \%$ (tabla

\section{Tabla 1}

Electorado, resultados y abstencionismo del referéndum sobre el tratado de libre comercio entre República Dominicana, Centroamérica y Estados Unidos en centros penitenciarios 2007

\begin{tabular}{c|c|c|c|c|c|c}
\hline País & Electorado & $\begin{array}{c}\text { Votos } \\
\text { emitidos }\end{array}$ & Sí & No & Participación & Abstencionismo \\
\hline Costa Rica & 5580 & 1584 & $52 \%$ & $48 \%$ & $28 \%$ & $72 \%$ \\
\hline
\end{tabular}

Fuente: TSE (2007).

Los datos de la tabla 1, reflejan una baja participación electoral en el contexto de los centros penitenciarios en Costa Rica. Esto podría apuntar que en el caso de formas directas de participación ciudadana y de alta complejidad política, se presume que no existió por parte de los sectores políticos involucrados la intención de incluir a la población privada de libertad dentro del proceso deliberativo, en el acceso a la información y el debate público.

\subsection{Recursos de amparo electoral}

Los procesos electorales dentro de las cárceles tienen el respaldo del Tribunal Supremo de Elecciones para que se realicen con las mismas condiciones que el resto de los ciudadanos costarricenses. Para dichos efectos, se cuenta con el recurso jurídico de los amparos electorales, que buscan proteger el acceso a los derechos político-electorales. En el 2014, se estableció que el Ministerio de Justicia deberá garantizar "que los privados de libertad ejerzan su derecho al voto, aunque sean cambiados de cárcel después de cerrado el padrón electoral" (Diario la Nación, 2014).

Lo anterior se debió a un recurso de amparo electoral interpuesto por la directora de la Defensa Pública Marta Iris Muñoz, posterior al primer proceso electoral presidencial y legislativo del año 2014 en favor de siete personas privadas de libertad que estimaron su derecho al sufragio dado que, por encontrarse privados de libertad, solicitaron su inscripción electoral en el Centro de Atención correspondiente; sin embargo, previo a las elecciones fueron trasladadas a un centro penitenciario diferente a aquel en que se encontraban empadronados, lo cual les impidió que votaran el 2 de febrero del 2014; además, señalaron que, de mantenerse ese traslado, tampoco podrán sufragar en la segunda ronda. 
Este recurso fue declarado sin lugar por los Magistrados del TSE por que el Ministerio de Justicia y Paz tiene la potestad de trasladar a la población privada de libertad a los centros donde considere más conveniente para el cumplimiento de su pena. Aun así, estableció una serie de obligaciones al Ministerio de Justicia para que realice todos los trámites pertinentes en atención a la solicitud de cambios en el padrón electoral, lo cual garantice el acceso a la participación política electoral.

Durante los últimos diez años, las personas privadas de libertad han aumentado el uso de este tipo de mecanismos para solucionar las ineficiencias de la institucionalidad relacionadas con la limitación del sufragio. El primer recurso que se encuentra data del proceso electoral nacional del año 2006 presentado por Álvaro Salas Torres (Voto n ${ }^{0}$ 394- E- 2006). En el referéndum nacional del 2007 se realizaron dos recursos de amparo electoral por este tema (votos 2760-E-2007 y 3326-E-2007), en el proceso electoral presidencial y legislativo 2014 se realizó uno (voto 1967- E1-2014) y en el año 2016 durante el proceso electoral municipal se realizó otra (voto 2016-94). Por su parte, también existen mecanismos no institucionalizados por los cuales las personas privadas de libertad exponen sus denuncias, como el caso de los medios de comunicación escrita.

\section{Conclusiones: privación de libertad y ejercicio de la ciudadanía}

La mayor parte de la población privada de libertad es vulnerabilizada a partir de desigualdades estructurales que determinan su condición (Oficina del Alto Comisionado de las Naciones Unidas para los Derechos Humanos (HCHR), p. 27, 2006). Este enfoque permite comprender la cuestión penitenciaria y la cárcel, a partir de las condiciones del medio social que la generan, lo que necesariamente tendrá un impacto en el ejercicio de la participación ciudadana.

Los indicadores muestran un bajo nivel de participación electoral, además de la ausencia de candidaturas de personas privadas de libertad a puestos de representación popular y la inexistencia de iniciativas de ley ciudadanas presentadas por población privada de libertad. Es decir, existen limitadas capacidades institucionales para hacer valer los derechos políticos formales de la participación ciudadana de las personas privadas de libertad, lo cual tiene un impacto dentro del sistema democrático en el tanto se excluye de la representación a una de las poblaciones más vulnerables del conglomerado social.

Existen otras iniciativas como la producción de actividades académicas mediante el debate realizado por el Centro de Investigación y Estudios Políticos (CIEP) y la 
Vicerrectoría de Acción Social de la Universidad de Costa Rica en el 2017. Estas iniciativas forman un precedente histórico (Murillo, 2017) en el fortalecimiento de la ciudadanía de las personas privadas de libertad, en lo que se refiere a informarse y opinar sobre asuntos públicos, además de pedir y obtener información de actores institucionales (Alfaro, 2017).

La evidencia mostrada permite concluir que se requieren condiciones que puedan propiciar un reconocimiento más amplio de las garantías necesarias para hacer efectivos los derechos políticos de esta población, no solo de sectores académicos sino de todo el sistema. Es fundamental no solo el acceso a la información, sino en las condiciones que se requieren para hacer efectivo el derecho a participar en actividades políticas a nivel interno, mediante una mejor coordinación institucional para el empadronamiento y traslado, entre otras.

Por eso, es necesario que se considere al Estado como ente jurídico que simultáneamente sanciona la privación de libertad y que debe garantizar en términos formales derechos como los asociados a la participación ciudadana en su sentido estricto. Esta contradicción pone en cuestión el uso del concepto de participación ciudadana clásico, pensado a partir de poblaciones que no son privadas de libertad. El imperativo garantista que sitúa a la persona privada de libertad como usuaria de todos sus derechos, excepto de su libertad, se desploma si consideramos que es exactamente la libertad la que permite hacer uso de una gran cantidad de derechos y contraer deberes relacionados con la participación ciudadana.

\section{Referencias}

Alfaro, J. (2017). UCR y Justicia llevarán debate presidencial a centro penitenciario. Semanario Universidad. Recuperado de: https://semanariouniversidad.com/pais/ ucr-justicia-llevaran-debate-presidencial-centro-penitenciario/

Asamblea Legislativa de la República de Costa Rica. (1998). Código Municipal. Ley n ${ }^{\circ}$ 7794. San José, Costa Rica.

Castro, M. y Sibaja, G. (2016). Participación ciudadana en democracia: espacios y mecanismos. San José, Costa Rica: Instituto de Formación y Estudios en Democracia, Tribunal Supremo de Elecciones.

Comisión Interamericana de Derechos Humanos (CIDH). (2008). Principios y Buenas Prácticas sobre la Protección de las Personas Privadas de Libertad en las Américas. Recuperado de: http://www.oas.org/es/cidh/mandato/Basicos/PrincipiosPPL.asp 
Diario la Nación. (2014). Justicia debe garantizar que reos ejerzan su derecho al voto aunque sean cambiados de cárcel. La Nación. Recuperado de: ww.nacion.com/ el-pais/politica/justicia-debe-garantizar-que-reos-ejerzan-su-derecho-al-voto-aunque-sean-cambiados-de-carcel/GPP5T7YSF5HWLPKHJYPPZHVTBQ/ story/

Ferrari, V. (2002). Derecho y sociedad. Elementos de sociología del derecho. Bogotá, Colombia: Universidad Externado de Colombia.

Focault, M. (1991). Vigilar y castigar. México: Siglo XXI.

Font, J., Blanco, I., Gomá, R. y Jarque, M. (2012). Mecanismos de participación ciudadana en la toma de decisiones locales: una visión panorámica. Revista Transformación, (50), 102-129.

Haas, P. (1992). Introduction: Epistemic Communities and International Policy Coordination. International Organization, 46 (1), 1-35

Herrera, J. (2008). Reinventando los Derechos Humanos. Andalucía: Atrapasueños.

Instituto Formación y Estudios en Democracia. (2016). Participación ciudadana en democracia: Espacios y mecanismos. San José: Tribunal Supremo de Elecciones.

Instituto Interamericano de Derechos Humanos (IIDH). (2011). Un acercamiento a la participación política desde la dimensión de la pobreza. San José, Costa Rica.

Londoño, M. (2007). Causas de la privación de libertad y perfil de la población penitenciaria. Recuperado de: http://repositorio.conare.ac.cr/bitstream/handle/20.500.12337/7728/CAP_7_\%20segundo_justicia\%202017. pdf? sequence $=1 \&$ isAllowed $=\mathrm{y}$.

Lutz, B. (2013). Lectura crítica: Castigar a los pobres: el gobierno neoliberal de la inseguridad social. Espiral, Estudios sobre Estado y Sociedad, (57), 177-189.

Ministerio de Justicia y Paz. (2017). Anuario Estadístico 2017. San José, Costa Rica: Departamento de Investigación y Estadística.

Ministerio de Justicia y Paz. (2017). Informe Proceso Electoral Carcelario 2014. San José, Costa Rica: Departamento de Investigación y Estadística.

Mouffe, C. (1999). El retorno de lo político. Comunidad, ciudadanía, pluralismo, democracia radical. Buenos Aires: Ediciones Paidós Ibérica.

Murillo, A. (2017). Presos acogieron debate presidencial: "siéntanse como en su casa". Semanario Universidad. Recuperado de: https://semanariouniversidad.com/pais/ candidatos-presidenciales-la-carcel-sientanse-casa/ 
Oficina del Alto Comisionado de las Naciones Unidas para los Derechos Humanos. (2006). Personas Privadas de Libertad, Jurisprudencia y Doctrina. Recuperado de: http://www.corteidh.or.cr/tablas/24430.pdf

Organización de las Naciones (ONU). (2015). Reglas Mínimas de las Naciones Unidas para el Tratamiento de los Reclusos. Nueva York. Recuperado de: http://www. un.org/ga/search/view_doc.asp?symbol=A/C.3/70/L.3\&referer=http://www. un.org/en/ga/third/70/proposalstatus.shtml\&Lang $=\mathrm{S}$

Organización de las Naciones Unidas (ONU). (1984). Convención contra la Tortura y Otros Tratos o Penas Crueles, Inhumanos o Degradantes. Nueva York. Recuperado de: http://www.ohchr.org/SP/ProfessionalInterest/Pages/CAT.aspx

Organización de los Estados Americanos (OEA). (2018). Derechos de las Personas Privadas de Libertad. Recuperado de: http://www.oas.org/es/cidh/ppl/default.asp

Pignataro, A. y Cascante, M. (2018). Los electorados de la democracia costarricense. Recuperado de: http://www.tse.go.cr/pdf/publicaciones/los-electorados-de-la-democracia-costarricense.pdf

Programa Estado de la Nación. (2017). II Informe Estado de la Justicia. San José. Recuperado de: https://www.estadonacion.or.cr/files/biblioteca_virtual/justicia/COMPLETO-2017.pdf

Sobrado, L. (2006). Experiencia costarricense del voto de Personas Privadas de Libertad. Revista Electoral del Tribunal Supremo de Elecciones, (3), 1-22.

Tribunal Supremo de Elecciones. (2007). Tratado de Libre Comercio República Dominicana- Centramérica- Estados Unidos. Recuperado de http://www.tse.go.cr/estadisticas_elecciones.htm

Tribunal Supremo de Elecciones. (2018). Datos electorales en centros penitenciarios 2002-2018. Recuperado de: https://www.tse.go.cr/estadisticas_elecciones.htm

Vargas, J. (2008). Perspectivas del institucionalismo y neoinstitucionalismo. Revista Ciencia Administrativa, (1), 47-58. 